\title{
A micropulse eye-safe all-fiber molecular backscatter coherent temperature lidar
}

\author{
${ }^{*}$ Cyrus F. Abari ${ }^{1,3,4}$, Xinzhao Chu ${ }^{1,2}$, Jakob Mann $^{4}$, and Scott Spuler $^{3}$ \\ ${ }^{1}$ Cooperative Institute for Research in Environmental Sciences, University of Colorado, Boulder, Colorado, USA \\ ${ }^{2}$ Department of Aerospace Engineering Sciences, University of Colorado Boulder, Boulder, Colorado, USA \\ ${ }^{3}$ National Center for Atmospheric Research, Earth Observing Lab, Boulder, CO, USA \\ ${ }^{4}$ Department of Wind Energy, Technical University of Denmark, Roskilde, Denmark \\ *Email: cyrus.abari@gmail.com
}

\begin{abstract}
In this paper, we analyze the performance of an all-fiber, micropulse, $1.5 \mu \mathrm{m}$ coherent lidar for remote sensing of atmospheric temperature. The proposed system benefits from the recent advances in optics/electronics technology, especially an all-fiber image-reject homodyne receiver, where a high resolution spectrum in the baseband can be acquired. Due to the presence of a structured spectra resulting from the spontaneous Rayleigh-Brillouine scattering, associated with the relevant operating regimes, an accurate estimation of the temperature can be carried out. One of the main advantages of this system is the removal of the contaminating Mie backscatter signal by electronic filters at the baseband (before signal conditioning and amplification). The paper presents the basic concepts as well as a Monte-Carlo system simulation as the proof of concept.
\end{abstract}

\section{INTRODUCTION}

Continuous high-resolution observation of atmospheric temperature profile in the troposphere is crucial for improved weather forecasting at the mesoscale [1]. In wind energy industry, where the latest turbines have reached heights beyond $200 \mathrm{~m}$, remote sensing of temperature has become compelling. At these heights, wind shear, veer, and turbulence are highly sensitive to the stratification due to temperature [2]. Furthermore, profiling horizontal variations of temperature is important for the flow characterization on varying terrain.

Lidars can provide high spatial and temporal resolution monitoring of thermodynamic variables in the atmosphere. The majority of existing temperature measurement lidars benefit from the direct detection principle. In these systems sub-micron wavelengths are employed to take advantage of a stronger Rayleigh backscatter, where $\beta \propto \lambda^{-4}$ ( $\beta$ is the molecular backscatter cross-section and $\lambda$ is the wavelength). There are a few disadvantages associated with such systems, e.g., they require highpower lasers which can be costly, bulky, and require high-maintenance. The transceivers in such systems can also be more costly and complicated to design and implement.
Coherent Doppler lidars (CDL), relying on Mie scattering, have been successfully used for remote sensing of wind [3], [4], [5]. Recent advances in fiber-optic technology and electronics have paved the way for a wide range of robust, compact, and cost-effective lasers and fiber-optic components. Successful realization of all-fiber CDLs operating at $1.5 \mu \mathrm{m}$ wavelength has already been well established, e.g., see [4], [6]. Although the existing CDLs rely on Mie scattering to capture the wind-induced Doppler spectrum, modified systems should be capable of recording the thermally broadened (Doppler) spectrum from the molecular backscatter signal [7].

Historically, a few pitfalls have prevented the realization of a molecular coherent temperature lidar (CTL). The Rayleigh backscatter signal is weak and has a thermally broadened Doppler spectrum with a relatively wide bandwidth $(\mathrm{BW})$, when compared to the narrowband Mie backscatter. The BW scales inversely with $\lambda$ and is outside the detection BW of the available photodetector (PD) technology for sub-micron wavelengths. Only recently, PDs and fast analogue to digital converters (ADC) have become available with detection BWs that are comparable with the BW of the Rayleigh backscatter Doppler signal (for longer wavelengths in the infrared region such as $1.5 \mu \mathrm{m})$. Optical image-reject downconversion of Doppler signals to baseband, essential for resolving positive and negative frequency shifts, has not been practically demonstrated, viz., not until recently [8]. This is an essential part of the proposed CTL to circumvent the restrictions imposed by the available BW of ADCs and PDs. The alternative, and conventionally employed, down-conversion to intermediate frequency (IF) band, places unrealistic requirements on the BW of the PDs and ADCs. A successful prototyping of an allfiber image-reject homodyne receiver demonstrated by Abari et al. [8] facilitates the realization of a molecular backscatter CTL as suggested in this paper. 


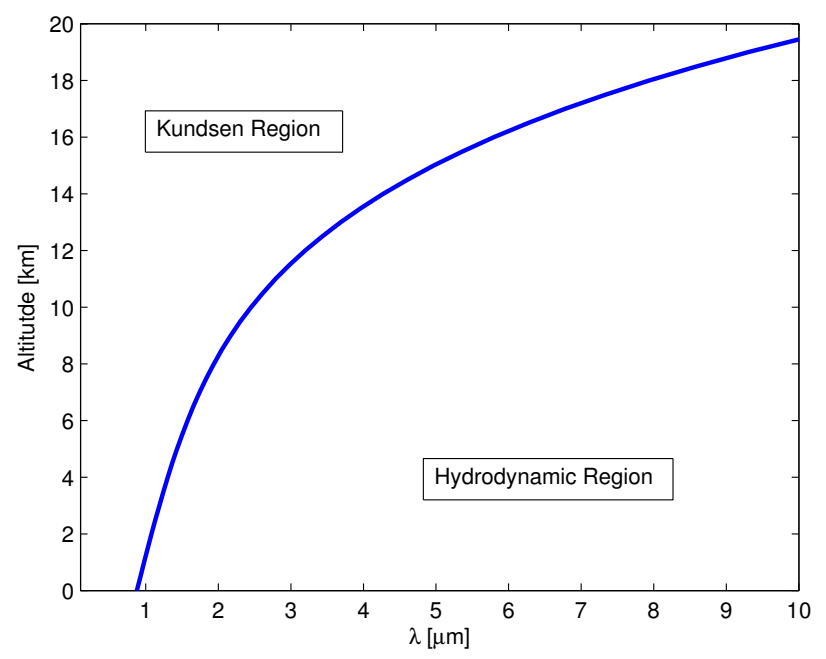

Fig. 1: Transition from the Kundsen to hydrodynamic region [7]. The region below the curve represents the area where the wavelength is longer than the mean free-path in the atmosphere, hence, SRB backscattering.

\section{SPONTANEOUS RAYLEIGH-BRILLOUIN (SRB) BACKSCATTERING AND SYSTEM DESCRIPTION}

A theoretical feasibility study of a molecular backscatter CTL was carried out by Rye [7] for a few wavelengths (i.e., 350, 2100, and $10600 \mathrm{~nm}$ ). The results indicate that the longer wavelengths, in the infrared (IR) spectrum, may operate in the hydrodynamic region, where $\lambda$ is larger than the mean free path in the air [9], [7]. In this region the return signal is not purely Rayleigh; The return spectra has contributions from the Rayleigh and spontaneous Brillouin backscatter signal, known as spontaneous Rayleigh-Brillouin (SRB), resulting in a triplet of peaks in the spectra. Fig. 1 shows the transition from the Kundsen (where the backscatter signal is largely dominated by the Rayeligh backscatter) to hydrodynamic region for different wavelengths and altitudes. As a result, due to the presence of a structured SRB backscatter Doppler spectrum in the hydrodynamic region [10] as well as the inherent CTL detection sensitivity, accurate estimation of temperature from a weak molecular backscatter is possible; The presence of narrowband Brillouin peaks provide a reliable measure of the atmospheric temperature [7]. As we will see in Sec. III, we have resorted to a least squares (LS) curve fitting between the estimated and expected spectra to estimate the temperature. The added benefit of LS curve fitting is the potential for extracting other parameters (e.g., atmospheric pressure) from the estimated spectra [7].

Following Tenti's S6 theory [9], the spectra associated with the SRB backscatter for $\lambda=1560 \mathrm{~nm}$ (at two

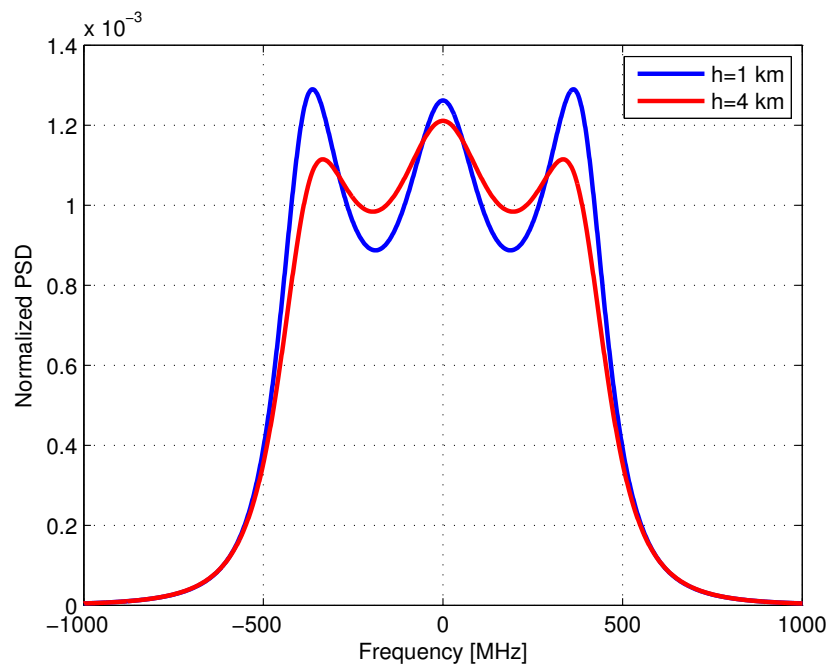

Fig. 2: SRB spectrum for $\lambda=1560 \mathrm{~nm}$ at two different altitudes in reference to sea level. The data is taken from the U.S. Standard Atmosphere Model.

different heights in reference to sea level) has been calculated and shown in Fig. 2. As the atmospheric pressure decreases (i.e., the altitude increases) the contribution from the Brillouin effect is reduced (as the mean free-path between the molecules becomes comparable with the laser wavelength) and the Rayleigh backscatter becomes more dominant. This is why the Brillouin peaks associated with the estimated spectra at an altitude of $h=4 \mathrm{~km}$ shown in Fig 2 are less pronounced when compared to the spectra estimated at $h=1 \mathrm{~km}$.

In light of Rye's [7] results, we have shown that it is possible to use a micropulse $1.5 \mu \mathrm{m}$ CTL to carry out an accurate remote sensing of temperature in the lower atmosphere. Although the micropulse system cannot rival the solid-state or gas-based IR laser, in terms of the maximum available pulse energy, it provides comparable average output powers. In other words, the integration time is competitive with the high power IR systems analyzed in [7]. High-quality all-fiber $1.5 \mu \mathrm{m}$ pulsed erbium doped fiber amplifiers (EDFA) with a PRR of $10-20 \mathrm{kHz}$ and pulse energy of $110-220 \mu \mathrm{J}$ (average optical power of $2.2 \mathrm{~W}$ ) are available as off-the-shelf components [11] and have been successfully integrated in commercially available all-fiber wind CDLs [6]. More powerful multi-stage EDFAs, benefiting from large core optical fibers, can be developed and integrated into the micropulse system to improve the range and required integration time.

Fig. 3 shows the schematic of the proposed all-fiber CTL. This system benefits from an all-fiber image-reject homodyne receiver [8] which allows the translation of the frequency-shifted signals into the baseband. Due to a relatively narrow BW associated with the 1560 


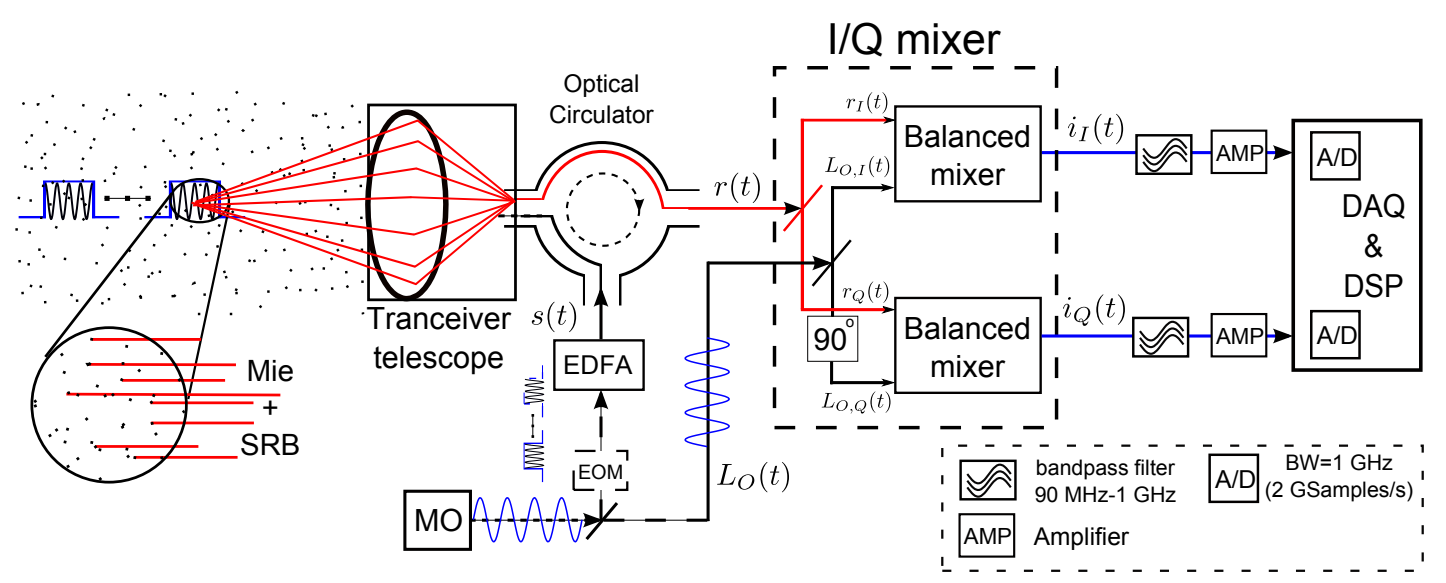

Fig. 3: An all-fiber pulsed image-reject homodybe CTL. The master oscillator (MO) provides a highly stable and narrow linewidth laser which is split into (by a beam splitter) a local oscillator (LO) and input signal to the electro-optic modulator (EOM). The EOM can be programmed to generate the desired pulse train which is amplified by the EDFA. The optical circulator isolates the transmit and receive signals. The in-phase/quadrature-phase (I/Q) mixer translates the spectral information into baseband for further processing. For a detailed analysis of the image-reject homodyne receiver please refer to Abari, et al. [8]. The bandpass filters, designed for a maximum expected mean wind speed, filter out the Mie signal.

$\mathrm{nm}$ molecular backscatter signal, a PD with a BW of $1 \mathrm{GHz}$ and two ADCs with a sampling rate of 2 GSamples/s provide the opportunity to resolve the spectral components, which upon further processing and digital signal processing allow the estimation of the relevant parameters such as temperature. A common problem associated with the detection and characterization of the molecular backscatter signal in the lower atmosphere is a strong contamination resulting from the Mie backscattering which dominates the return signal. In direct detection techniques, such as high spectral resolution lidars (HSRL), optical filters, only available for a limited number of wavelengths, are employed to filter out the Mie component from the return signal. In the proposed system, we suggest the removal of the Mie backscatter directly at the output of the PD. If the dominant Mie component is not removed immediately before amplification and conditioning of the detected signal, it will either saturate or occupy the dynamic range of the following stages, i.e., the electronic components adopted for signal amplification, conditioning, and sampling. One of the main merits of this system is to remove the Mie component through reliable, cost-effective, and accurate electronic filters. In this system the electronic filter BW can be designed for the expected maximum mean wind speed. Moreover, due to the nature of coherent detection, background light removal, necessary in direct detection systems, is not required in the proposed system.

\section{Simulation Results}

The performance of the system suggested in this paper is evaluated through modeling and Monte-Carlo

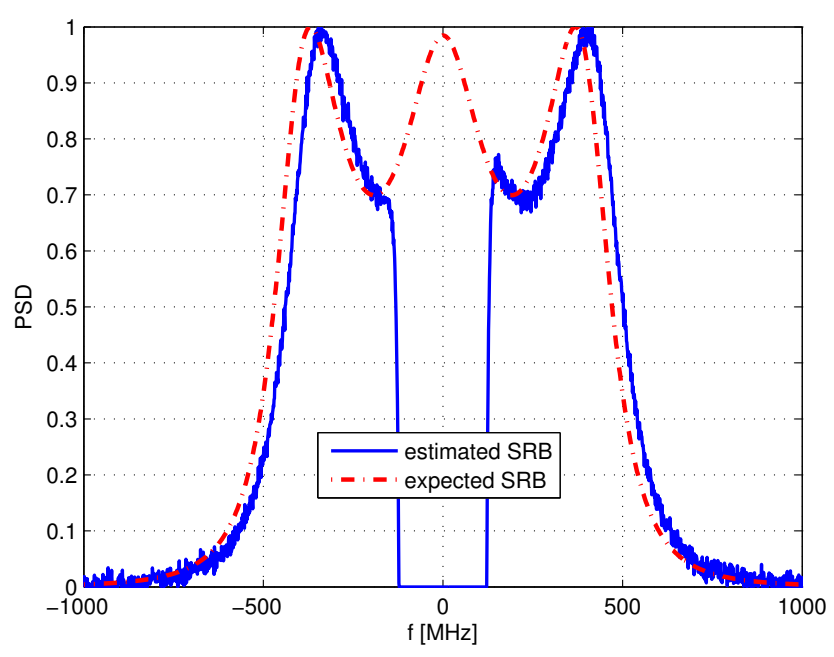

Fig. 4: The simulated return spectrum (target range of $1 \mathrm{~km}$, please see Table I). The observed mean frequency shift in the blue curve is due to a presumed mean wind speed of $25 \mathrm{~ms}^{-1}$. simulations performed in Matlab. In our simulation the combined effects of Mie and molecular backscattering are taken into account. Since the aerosol type and density in the atmosphere is hard to predict and model, we have assumed a moderate loading of aerosols [12] with uniform scattering throughout the measurement range. The Rayleigh backscatter parameters have been adopted from the U.S. Standard Atmospheric Model. Furthermore, the Monte-Carlo simulations have been done for a CTL located $1 \mathrm{~km}$ above the sea level performing a horizontal scan. The simulation parameters are listed in Table I. Please note that $\beta_{\text {Mie }}$ and $D$ represent Mie volume backscatter coefficient and the telescope diameter, respectively. 
TABLE I: Simulation parameters

\begin{tabular}{|l|c||l|c|}
\hline$E_{t}[\mu \mathrm{J}]$ & 220 & PRR $[\mathrm{kHz}]$ & 10 \\
\hline PD BW $[\mathrm{GHz}]$ & 1 & $f_{s}[\mathrm{GHz}]$ & 2 \\
\hline pulse length $[\mathrm{ns}]$ & 400 & resolution $[\mathrm{m}]$ & 95 \\
\hline integration time $[\mathrm{s}]$ & 60 & $\mathrm{~N}[\mathrm{FFT}$ points $]$ & 1024 \\
\hline$D[\mathrm{~cm}]$ & 20 & $\beta_{\text {Mie }}\left[\mathrm{m}^{-1} \mathrm{sr}^{-1}\right]$ & $1.83 \times 10^{-7}$ \\
\hline
\end{tabular}

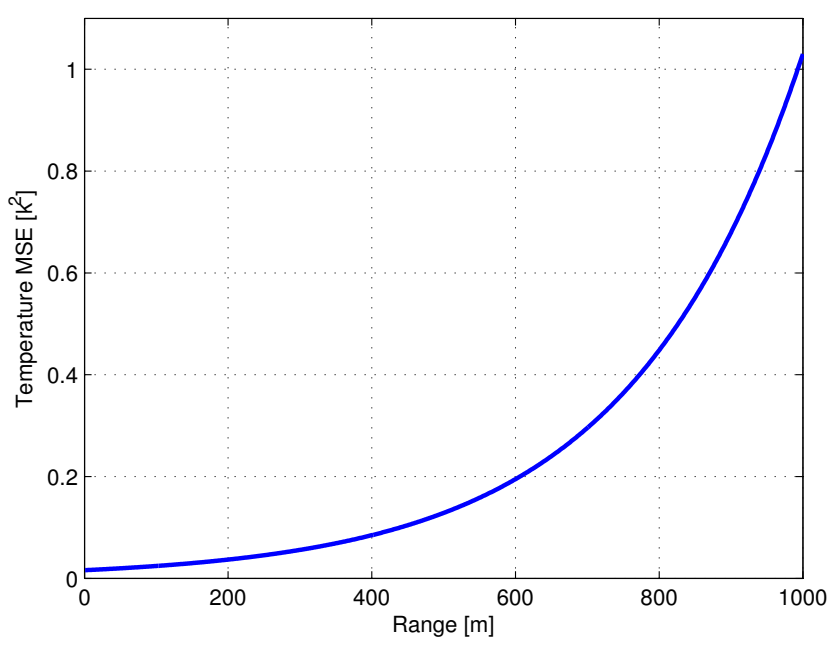

Fig. 5: MSE (variance) of the temperature estimator (for a temporal resolution of $60 \mathrm{~s}$ ) versus target range.

For the estimation of the temperature we have resorted to an LS estimator in this paper. The estimator employs a LS fitting of the acquired spectra; An iterative procedure where the SRB curve resulting in the LS error between the acquired spectra and the curve is selected as the best estimate. For the performance characterization of our system, we have adopted the mean square error (MSE) of the temperature estimator for different ranges; The MSE can be viewed as the variance of the estimator. Fig. 5 shows the MSE of the temperature estimator adopted in this paper for different ranges. As we can see the estimator provides an estimate of the temperature with a variance better than $1 \mathrm{~K}$ for a measurement range of up to $1 \mathrm{~km}$. It can be shown that the measurement range can be improved by increasing the pulse energy or integration time. Due to the availability of high speed computers and digital signal processors, more advanced signal processing can be adopted to improve the temperature estimation accuracy which can result in a longer measurement range for a given system configuration and atmospheric condition.

\section{CONCLUSIONS}

In this paper, we have shown that in light of recent advances in fiber-optic and electronic technology it is feasible to build a compact and robust coherent lidar for remote sensing of atmospheric temperature. Compared to other lidars, the proposed system should be lowcost, low-maintenance, and can be deployed as an eyesafe system (in the same fashion $1.5 \mu \mathrm{m}$ wind Doppler lidars with similar optical output powers operate, e.g., a scanning CTL). Due to it's form factor the system can be deployed as ground-base or airborne. Furthermore, the system has the potential to provide simultaneous measurements of wind and pressure (besides temperature); This will be investigated in our future work. We have adopted only one simple estimator, i.e., LS, to demonstrate the feasibility of the proposed CTL. However, we believe there is room for improved estimation algorithms where the Cramer-Rao lower bound (CRLB), computationally calculated in [7], can be achieved.

\section{REFERENCES}

[1] Committee on Developing Mesoscale Meteorological Observational Capabilities to Meet Multiple Needs, National Research Council, Observing Weather and Climate from the Ground Up: A Nationwide Network of Networks. Washington, D.C.: The National Academies Press, 2009.

[2] A. Sathe, J. Mann, T. Barlas, W. Bierbooms, and G. van Bussel, "Influence of atmospheric stability on wind turbine loads," Wind Energy, vol. 16, no. 7, pp. 1013-1032, 2013.

[3] F. Hall, R. Huffaker, R. Hardesty, M. Jackson, T. Lawrence, M. Post, R. Richter, and B. Weber, "Wind measurement accuracy of the NOAA pulsed infrared Doppler lidar," Appl. Opt., vol. 23, no. 15, pp. 2503-2506, 1984.

[4] S. M. Spuler, D. Richter, M. P. Spowart, and K. Rieken, "Optical fiber-based laser remote sensor for airborne measurement of wind velocity and turbulence," Appl. Opt., vol. 50, pp. 842851, 2011.

[5] R. M. Huffaker and R. M. Hardesty, "Remote sensing of atmospheric wind velocities using solid-state and $\mathrm{CO}_{2}$ coherent laser systems," Proc. IEEE, vol. 84, pp. 181-204, 1996.

[6] WINDCUBE V2, a 200m vertical wind Doppler lidar. [Online]. Available: http://www.leosphere.com/products/verticalprofiling/windcube-v2. [Accessed Feb. 6, 2015].

[7] B. J. Rye, "Molecular backscatter heterodyne lidar: a computational evaluation," Appl. Opt., vol. 37, no. 27, pp. 6321-6328, 1998.

[8] C. F. Abari, A. T. Pedersen, and J. Mann, "An all-fiber imagereject homodyne coherent Doppler wind lidar," Opt. Express, vol. 22, pp. 25 880-25 894, 2014.

[9] C. D. Boley, R. C. Desai, and G. Tenti, "Kinetic models and Brillouin scattering in a Molecular Gas," Canadian Journal of Physics, vol. 50, no. 18, pp. 2158-2173, 1972.

[10] Z. Gu, B. Witschas, W. van de Water, and W. Ubachs, "Rayleigh-Brillouin scattering profiles of air at different temperatures and pressures," Appl. Opt., vol. 52, no. 19, pp. 46404651, 2013.

[11] $1.5 \mu \mathrm{m}$ LONG PULSE FIBER AMPLIFIER, (Keopsys, 2015). [Online]. Available: http://www.keopsys.com

[12] V. Srivastava, J. Rothermel, A. D. Clarke, J. D. Spinhirne, R. T. Menzies, D. R. Cutten, M. A. Jarzembski, D. A. Bowdle, and E. W. McCaul, "Wavelength dependence of backscatter by use of aerosol microphysics and lidar data sets: application to 2.1$\mu \mathrm{m}$ wavelength for space-based and airborne lidars," Appl. Opt., vol. 40, pp. 4759-4769, 2001. 